\title{
A Search for Elliptic Curves With Large Rank
}

\author{
By David E. Penney and Carl Pomerance
}

\begin{abstract}
A search procedure is described for finding elliptic curves with rational coefficients for which the group of rational points has large rank. Specific examples are given of elliptic curves with rank $\geqq 6$.
\end{abstract}

We recall Mordell's famous theorem that the set of rational points on a genus 1 curve with rational coefficients forms a finitely generated abelian group. The rank of such a curve is defined to be the number of free generators of this group. Wiman [3] gave the curve

$$
y^{2}=x^{3}+338 x^{2}+13432 x
$$

as an example of a rank 4 elliptic curve. (Wiman claims the curve (1) has rank 6 , but this is because his definition of rank is the minimum number of generators of the group of rational points, including points of finite order.)

Néron [1] proved that rank 10 elliptic curves exist, but gave no examples. In fact, no specific examples of elliptic curves of rank exceeding 4 have been published.

In this paper, we describe a procedure which computerizes a search for large rank elliptic curves. In particular, we give several examples of curves with rank $\geqq$ 6. In a future paper, we hope to give examples with even larger rank.

We restrict our attention to curves of the form

$$
y^{2}=x^{3}+a x^{2}+b x
$$

where $a, b \in \mathbf{Z}$ and $a^{2}-4 b$ is not a square. (We note that Wiman's example (1) is not in this form, since $338^{2}-4 \cdot 13432=246^{2}$.) Let $\Gamma$ be the group of rational points on (2), where the identity of $\Gamma$ is 0 , the point at infinity.

Since $\Gamma$ is a finitely generated abelian group, we write $\Gamma \cong \mathbf{Z}^{r} \oplus \mathbf{Z}_{p_{1} a_{1}} \oplus \cdots \oplus \mathbf{Z}_{p_{k} a_{k}}$ where $p_{1}, \ldots, p_{k}$ are primes and $r$ is the rank of $\Gamma$. Since $a^{2}-4 b$ in (2) is not a square, there is only one rational root to $x^{3}+a x^{2}+b x$; so $\Gamma$ has precisely one rational point of order 2 , namely $(0,0)$. Hence, precisely one of $p_{1}, \ldots, p_{k}$ is 2 . Then $\Gamma / 2 \Gamma \cong \mathbf{Z}_{2}^{r+1}$.

Now it is easy to show from the definition of doubling a point on $\Gamma$, that if $(x, y) \in 2 \Gamma$, then $x \in Q^{2}$; that is, $x$ is the square of a rational number. Denote by $Q^{*}$ the group of nonzero rationals, and let $\alpha: \Gamma \rightarrow Q^{*} / Q^{* 2}$ where $\alpha(x, y)=x Q^{* 2}$ if $x \neq 0, \alpha(0,0)=b Q^{* 2}$, and $\alpha(0)=Q^{* 2}$. It follows that $\alpha$ is a group homomorphism (for example, see Tate [2]) and that $2 \Gamma \subset \operatorname{ker} \alpha$.

Summing up, we have $2^{r+1}=o\left(\mathbf{Z}_{2}^{r+1}\right)=o(\Gamma / 2 \Gamma) \geqq o(\Gamma / \operatorname{ker} \alpha)=o(\operatorname{im} \alpha)$. Hence, if we could compute $o(\operatorname{im} \alpha)$, we would have a lower bound for the rank $r$ of $\Gamma$. A proof of the following theorem may be found in Tate [2].

Received October 4, 1973.

AMS (MOS) subject classifications (1970). Primary 10B10; Secondary 14G25, 14H30.

Key words and phrases. The rank of an elliptic curve.

Copyright @ 1974, American Mathematical Society 
THEOREM. im $\alpha=\left\{b Q^{* 2}\right\} \cup\left\{n Q^{* 2}: n \in \mathbf{Z}, n \mid b\right.$, and $n u^{4}+b v^{4} / n+a u^{2} v^{2}=w^{2}$ has a solution $(u, v, w)$ in pairwise prime nonzero integers $\}$.

The quartic mentioned in the theorem is of ten difficult to analyze, so we restrict our attention to those quartics which allow a solution with $u=v=1$. Namely, we study

$$
A=\left\{b Q^{* 2}\right\} \cup\left\{n Q^{* 2}: n \in \mathbf{Z}, n \mid b, n+b / n+a \text { is a nonzero square }\right\} .
$$

Let $B=$ the subgroup of $\operatorname{im} \alpha$ generated by $A$. Then $B$ is isomorphic to a subgroup of $\mathbf{Z}_{2}^{r+1}$ and hence $B \cong \mathbf{Z}_{2}^{s}$ for some $s \leqq r+1$. Hence, if we can compute $o(B)$ for a given choice of $a$ and $b$ in (2), we will have computed a lower bound for the rank $r$ of $\Gamma$.

For a given choice of $a$ and $b$ in (2), we ask the computer to determine the members of

$$
A^{\prime}=\{n: n \in \mathbf{Z}, n \mid b, n+b / n+a \text { is a square }\} .
$$

By examining this set, it is not hard to compute $A$ and then proceed to compute $o(B)$.

We give the following numerical example to illustrate the above process. Let $\Gamma$ be the group of rational points on $y^{2}=x^{3}+17 x^{2}-105 x$. It is a simple matter to compute $A^{\prime}$, the set defined in (4). Namely, $A^{\prime}=\{-21,-15,-7,-3,-1,5,7,15,35$, $105\}$. Then the subgroup $B$ of $\operatorname{im} \alpha$ is $\left\{n Q^{* 2}: n \in \mathbf{Z}, n \mid 105\right\}$ and $o(B)=16$. Hence, $y^{2}=x^{3}+17 x^{2}-105 x$ has rank $\geqq 3$.

In practice, we make a choice for $b$ in (2) and let the computer search for a "good" choice for $a$. Indeed, for a given $b$, the computer has the set $\{n+b / n: n \mid b\}$ stored as an increasing sequence. For each choice of $a$, the computer forms the set $\{n+b / n+a: n \mid b, n+b / n+a \geqq 0\}$. This last set is searched for squares. If we have hit upon a good choice for $a$, i.e., there are many (say $\geqq 4$ ) squares in the set, then the computer prints out $n, b / n, n+b / n+a$ whenever $n+b / n+a$ is a square. The computer identifies an integer $x \geqq 0$ as a square by first verifying that $x$ is a square for various small moduli, and if $x$ passes these tests, $\left[x^{1 / 2}\right]$ is found by Newton's method written in integer programming for speed. Then $x$ is a square if and only if $\left[x^{1 / 2}\right]^{2}=x$.

In (2), we specify that $a^{2}-4 b$ is not a square. However, for a given $b$, the computer might well choose an $a$ such that $a^{2}-4 b$ is a square. It is not necessary to patch up this "flaw" since $a^{2}-4 b$ is a square if and only if $x^{2}+a x+b=0$ has an integral root if and only if for some divisor $n$ of $b, n+b / n+a=0$. Hence, the square 0 appears on our printout if and only if $a^{2}-4 b$ is a square. Thus, we merely ignore those choices for $a$ and $b$ where the square 0 appears.

There are infinitely many ways of choosing $a$ and $b$ in (2). We need, therefore, a method of deciding which choices should be tried. Experience and a few elementary considerations, most of which we omit here, have led us to make the following restrictions on the choices for $a$ and $b$.

First, we assume $b$ is odd and square-free.

For a given $b$, we choose $a$ so that $0<a<f(b)<|b|$ where $f(b)$ is an arbitrarily chosen bound that increases with $|b|$. Furthermore, we take $a$ $\equiv 2(\bmod 3)$ and $a \equiv 0,2$, or $3(\bmod 5)$. If $b \equiv 3(\bmod 8)$, we choose $a$ $\equiv 5(\bmod 8)$. If $b \equiv 7(\bmod 8)$, we choose $a \equiv 1(\bmod 8)$. If $b \equiv 1(\bmod 4)$, we 
choose $a$ in a few (possibly 1) congruence classes mod 64. The following lemmas provide some explanation.

Lemma 1. If $b \equiv 3(\bmod 4)$, then $x+b x^{-1} \equiv 1+b(\bmod 8)$ for every odd $x$.

Proof. If $x$ is odd, then $x \equiv x^{-1}(\bmod 8)$, so $x+b x^{-1} \equiv x+b x \equiv x(1+b)$ $\equiv 1+b(\bmod 8)($ since $1+b \equiv 0(\bmod 4))$.

LEMMA 2. If $b \equiv 1(\bmod 4)$, then $x+b x^{-1}$ has precisely 4 values as $x$ ranges over the 32 odd congruence classes mod 64.

Proof. This lemma is easily verified by proving that for each odd $x \bmod 64$, there are precisely 8 odd $y$ 's mod 64 such that $x+b x^{-1} \equiv y+b y^{-1}(\bmod 64)$. In fact, this last congruence holds if and only if $(x-y)(b-x y) \equiv 0(\bmod 64)$ if and only if

(1) if $b \equiv 1(\bmod 8)$, then $y \equiv x(\bmod 8)$,

(2) if $b \equiv 5(\bmod 8)$, then $y \equiv x(\bmod 16)$ or $y \equiv x^{2}+b x-1(\bmod 16)$.

The following table sums up the highlights of what we found:

TABLE

\begin{tabular}{lrl}
\hline \multicolumn{1}{c}{$b$} & $a$ & rank \\
\hline & & \\
$3 \cdot 5 \cdot 13 \cdot 17 \cdot 29$ & 1217 & $\geqslant 5$ \\
$3 \cdot 5 \cdot 7 \cdot 11 \cdot 13 \cdot 17$ & 5513 & $\geqslant 5$ \\
$3 \cdot 5 \cdot 7 \cdot 11 \cdot 13 \cdot 17$ & 7265 & $\geqslant 5$ \\
$-3 \cdot 5 \cdot 7 \cdot 11 \cdot 13 \cdot 17$ & 29162 & $\geqslant 5$ \\
$3 \cdot 5 \cdot 7 \cdot 11 \cdot 13 \cdot 17 \cdot 19 \cdot 23$ & 53213 & $\geqslant 6$ \\
$3 \cdot 5 \cdot 7 \cdot 11 \cdot 13 \cdot 17 \cdot 19 \cdot 23$ & 80885 & $\geqslant 6$ \\
$3 \cdot 5 \cdot 7 \cdot 11 \cdot 13 \cdot 17 \cdot 19 \cdot 23$ & 100757 & $\geqslant 6$ \\
$-3 \cdot 5 \cdot 7 \cdot 11 \cdot 13 \cdot 17 \cdot 19 \cdot 23$ & 5858 & $\geqslant 6$ \\
$-3 \cdot 5 \cdot 7 \cdot 11 \cdot 13 \cdot 17 \cdot 19 \cdot 23$ & 47138 & $\geqslant 6$ \\
$-3 \cdot 5 \cdot 7 \cdot 11 \cdot 13 \cdot 17 \cdot 19 \cdot 23$ & 68258 & $\geqslant 6$ \\
$-3 \cdot 5 \cdot 7 \cdot 11 \cdot 13 \cdot 17 \cdot 19 \cdot 23$ & 74882 & $\geqslant 6$ \\
$-3 \cdot 5 \cdot 7 \cdot 11 \cdot 13 \cdot 17 \cdot 19 \cdot 23$ & 82658 & $\geqslant 6$ \\
$-3 \cdot 5 \cdot 7 \cdot 11 \cdot 13 \cdot 17 \cdot 19 \cdot 23$ & 93122 & $\geqslant 6$
\end{tabular}

Department of Mathematics

University of Georgia

Athens, Georgia 30602

1. A. NÉron, "Problèmes arithmétiques et géométriques rattachés à la notion de rang d'une courbe algébrique dans un corps," Bull. Soc. Math. France, v. 80, 1952, pp. 101-166. MR 15,151.

2. J. TATe, Rational Points on Elliptic Curves, Philips Lectures, Haverford College, 1961.

3. A. Wiman, "Über rationale Punkte auf Kurven dritter Ordnung vom Geschlechte Eins," Acta Math., v. 80, 1948, pp. 223-257. MR 10,472. 